\title{
An Hybrid Prediction-based Routing Approach for Reducing Routing Inaccuracy in Optical Transport Networks
}

\author{
Wilson Ramirez*, Xavier Masip-Bruin*, Eva Marín-Tordera*, Marcelo Yannuzzi ${ }^{\dagger}$, Anny Martinez ${ }^{*}$, Sergio \\ Sánchez-López*, Victor López ${ }^{\ddagger}$ \\ *Advanced Network Architectures Lab (CRAAX), Technical University of Catalonia (UPC), Spain \\ ${ }^{\dagger}$ Networking and Information Technology Lab (NetIT Lab) \\ Emails: \{wramirez, xmasip, eva, yannuzzi, annym, sergio\}@ac.upc.edu \\ $\ddagger_{\text {Telefonica I+D, Spain, vlopez@tid.es }}$
}

\begin{abstract}
The advent of network technologies such as Automatically Switched Optical Networks (ASON) and Generalized Multiprotocol Label Switching (GMPLS) pave the way to the deployment of flexible optical transport networks (OTNs). The flexibility of OTNs is a feature highly demanded in dynamic scenarios where lightpaths are continuously set up and torn down on a short-term basis. Unfortunately, the availability and accuracy of network state information in dynamic scenarios are both limited, causing a severe impact on both performance and scalability of Routing and Wavelength Assignment (RWA) algorithms. In this paper we devise a promising routing scheme so-called Hybrid Prediction-based Routing (HPBR). HPBR combines prediction strategies with an innovative method to select the most suitable routing metric, aiming at reducing both the dissemination of network state information and the blocking probability. Our findings validate that the proposed scheme significantly reduces the blocking probability compared with other routing schemes, while avoiding the need to periodically disseminate network state information.
\end{abstract}

Index Terms-Prediction Routing; Routing Inaccuracy

\section{INTRODUCTION}

Optical Transport Networks (OTNs) provide the flexibility and transmission capacity for suitable handling the skyrocketing demand of bandwidth required by future Internet applications [1]. One of the building blocks of OTNs is the Wavelength Division Multiplexing (WDM) technology. WDM allows a single optical fiber patch to simultaneously convey different wavelengths. To properly select a lightpath (both wavelength and path) Routing and Wavelength Assignment (RWA) algorithms jointly with control plane protocols such as Automatically Switched Optical Networks (ASON) and Generalized Multiprotocol Label Switching (GMPLS) are commonly used [2], [3].

The performance of an RWA algorithm is severely affected in dynamic scenarios where lightpaths are continuously setup and tear down on a short-term basis. This effect is mainly motivated by two issues: 1) the connection setup delay; and 2) the inaccuracy of the network state information [4]. In light of this, the study of RWA in the context of dynamic scenarios is gaining momentum in network research, motivated by the fact that nowadays internet applications such as Video on demand (VoD) or bulk transfer data, demand huge bandwidth and connectivity in an agile manner, which undoubtedly can overload the network with a high volume of connection requests (CRs).

On a source-based routing scenario-one of the ASON recommendations [5]-, the availability and accuracy of network state information have a profound impact on both performance and scalability of RWA algorithms. Indeed, inaccurate network state information might result in sub-optimal path selections that potentially lead to an increase of the blocking probability. The main factors of inaccurate network state information are infrequent information dissemination due to overhead issues and high propagation delays.

The rationale of this paper is to deal with the routing "inaccuracy problem". To this end, we propose a novel scheme so-called Hybrid Prediction-based Routing (HPBR). HPBR exploits both prediction techniques and an innovative method to select the most suitable routing metric, aiming at reducing the blocking probability without requiring network state information.

The rest of this paper is organized as follows. Section II delves into the state of the art on the routing inaccuracy problem. Section III introduces the proposed routing algorithm. Section IV describes the simulation model and the network scenarios used. Section V presents the obtained numerical results. Finally, Section VI provides final conclusions and future line of work.

\section{RELATED WORK}

The study of RWA algorithms has been widely studied over the years [6]. Nonetheless, only a small percentage of these studies take into account inaccurate network state information. In this section, we introduce in a nutshell several contributions specifically related to the routing inaccuracy problem.

The study presented in [7] is a pioneer work dealing with the routing inaccuracy problem, where authors focus on delay and bandwidth constraints applications, and propose probabilistic models to express the uncertainty of network state information. A similar study can be found in [8] where authors specifically focus on bandwidth constrained applications by means of a so-called Safety-Based routing. 
More recent works such as [9] propose a so-called BypassBased Routing, where authors claim to overcome the performance of the Safety-based Routing. The Bypass-Based Routing is based on a novel metric to model uncertainty combined with a mechanism that bypasses congested links -whenever a path selection is sub-optimal.

On the other hand, there are several studies available in the literature related to the routing inaccuracy problem in optical networks. The study in [10] extends the BBR algorithm for optical networks considering wavelength conversion. Whereas authors in [11] provide an analytical model to assess the performance of source-based routing in optical networks with inaccurate network state information. The study in [12] also addresses the routing inaccuracy problem in optical networks. The authors propose a parallel reservation scheme. Nevertheless, a handicap of this approach is its high consumption of network resources.

The aforementioned studies require some network state dissemination whatsoever -even thought this one is significantly reduced. It was the study presented in [13] which plunges into the routing inaccuracy problem on OTNs, by guarantying that the dissemination of network state information is restricted only to topological changes. To this end, authors propose the Predictive-Selection Routing algorithm (PSR) based on prediction branch techniques introduced in [14]. Basically, PSR relies on a two-bit counter to predict a route availability. On this basis, if a route is selected, but a certain connection cannot be provisioned along this route, its counter value is increased. Conversely, if the connection can be provisioned along a route, its route counter is decreased. It must be noticed that a two-bit counter is enough to keep historical behavior of routes. Otherwise, a low counter value is unable to properly model a route availability, whereas higher values add an enormous degree of hysteresis which causes sub-optimal paths selections -driven by inertia generated by a high counter value.

Motivated by the tradeoff between blocking probability and network state information achieved by PSR, the studies available in [15] and [16] also use the prediction strategies for OTN. On one hand, authors in [15] combine both BBR and PSR scheme- obtaining the so-called Balanced Vulnerable Predictive Path (BVP2)-scheme, aiming at improving the performance of PSR. On the other hand, authors in [16] propose a RWA scheme referred to as Fuzzy-based Routing (FRA), that not only combines both BBR and PSR, but it is also enriched with fuzzy based techniques to enhance the modeling of route availability.

In this paper, we propose a source-based routing scheme for addressing the routing inaccuracy problem in OTNs referred to as Hybrid Prediction-based Routing (HPBR). HPBR also exploits the use of prediction techniques but differs from the schemes proposed in [13], [15], [16] in several aspects.

First, the schemes proposed in [13-16] assume a two-bit counter (assigned locally by each node) for each route prediction counter per route. We adopt a finer granularity approach. To this end, each optical node keeps track of optical links availability by means of a two-bit counter -prediction counter per link. Second, we evaluate the proposed scheme in extended scenarios. Finally, HPBR is not bounded to a unique routing metric; it dynamically selects the most suitable metric according to the network scenario.

\section{THE HPBR ALGORITHM}

HPBR exploits the use of a two-bit counter to predict optical links availability. A counter value is increased or decreased as follows: $p_{i}^{(s)}=p_{i}^{(s)}+1 \Longleftrightarrow$ a connection along link $i$ is blocked and $p_{i}^{(s)}<3$; and $p_{i}^{(s)}=p_{i}^{(s)}-1 \Longleftrightarrow$ a connection along link $i$ can be successfully provisioned and $p_{i}^{(s)}>0$. Thereby, whenever a lightpath must be selected to provision a connection demand, the availability of a route is locally computed by each node $(s)$ as shown in Equation (1).

$$
\mathcal{L}_{j}^{1(s)}=\sum_{i \in j} v_{i}^{(s)} p_{i}^{(s)}
$$

As shown in Equation (1) $\mathcal{L}_{j}^{1(s)}$ is the availability of route $j$, $p_{i}^{(s)}$ and $v_{i}^{(s)}$ are the prediction counter and vulnerability of link $i$ respectively based on the network state information available on node $s$. A high value of $\mathcal{L}_{j}^{1(s)}$ means that route $j$ may be unavailable, the contrary occurs with low values.

The vulnerability degree $\left(v_{i}^{(s)}\right)$ is a concept introduced by [15] and used to model whether an optical link may lead to a connection blocking. The vulnerability degree of a link is computed as shown in Equation (2).

$$
v_{i}^{s}= \begin{cases}1, & \Longleftrightarrow 1-\frac{b_{r e q}}{b_{i}^{(s)}}<\epsilon \\ 0, & \text { otherwise }\end{cases}
$$

The bandwidth on link $i$, as well as its vulnerability, are locally determined by the source node, according to the node's previous allocations of resources along a route formed by link $i$, since HPBR minimizes the dissemination of network state information (recall the routing inaccuracy problem).

The vulnerability degree of a link is selected according to the parameter $\epsilon$, so-called blocking factor, which is a predefined threshold reflecting the degree of inaccuracy tolerated by HPBR. Thus, parameter $\epsilon$ must be properly set according to the network scenario, in order to model link availability inaccuracy in a precise manner. Indeed, the blocking factor modeling is left for future work.

In addition, notice that a prediction counter value is only considered for computing the route availability whenever the link is considered vulnerable. Therefore, even though the value of a prediction counter of a link is greater than zero, if this link is not vulnerable its prediction counter does not affect the availability of a route.

For the purpose of explaining the reasons that motivated us to adopt a finer granularity for the prediction counters, we consider the topology depicted in Fig. 1a, where source-based routing is assumed. A connection request $\left(C R_{1}\right)$ needs to be provisioned demanding the allocation of $4 \lambda$ with nodes $S$ and $D$ as source and destination respectively. As a consequence, route $S-1-2-D$ is selected, but it cannot be provisioned due to lack of bandwidth. This occurs because of the inaccuracy of the network state information of optical node $S$, which reflects $b_{1-2}^{(s)}$ with an available capacity of $8 \lambda$. However, the 


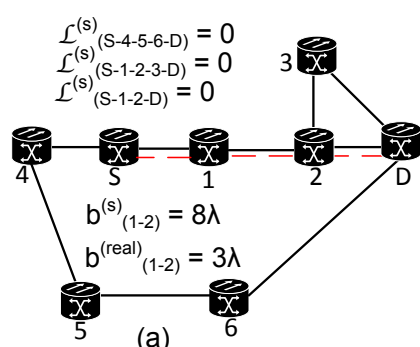

(a)

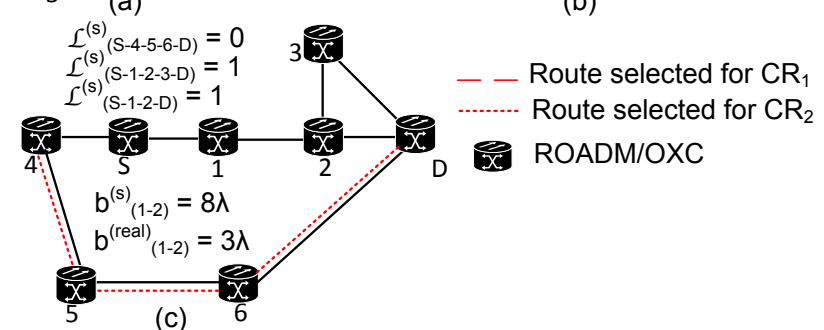

Figure 1. a) and b) High-granularity counters; c) Fine-granularity counters.

real bandwidth of link $1-2$ is $3 \lambda$, less than the bandwidth requested by $C R_{1}$, i.e., the state information stored in node $S$ is outdated. If prediction counters per route (high-granularity counters) are used, then the counter of route $S-1-2-D$ is increased.

Consider now the scenario depicted in Fig. 1b, where a second connection request $\left(C R_{2}\right)$ reaches node $S$ with similar characteristics ( $5 \lambda$ of bandwidth and nodes $S$ and $D$ as endpoints) as $C R_{1}$. As a result, node $S$ avoids selecting route $S-1-2-D$ (due to its counter value) and attempts to provision a connection along route $S-1-2-3-D$; however, $C R_{2}$ cannot be provisioned. This occurs because link $1-2$ does not have enough bandwidth to allocate $C R_{2}$, but a highgranularity counter does not capture the unavailability of link $1-2$; hence route $S-1-2-D$ is shown as available.

After carefully observing the scenarios depicted in Fig. 1a and Fig. 1b, an intuitive thought is to use fine-granularity counters. In light of this, take into account the scenario depicted in Fig. 1c. When $C R_{2}$ reaches node $S$ this node captures the unavailability of route $S-1-2-3-D$. This is because the counter of link $1-2$ (a link part of route $S-1-2-3-D)$ is not 0 ; hence, route $S-4-5-6-D$ is selected -all of the links forming this route have their counter values on 0 . By means of finer granularity counters the blocking probability is reduced, as we demonstrate and validate by the numerical results presented in Section V.

As such, HPBR uses fine-granularity counters, but it also uses two metrics for routing purposes. On one hand, the first metric, computed as shown in Equation (3), is an enhancement of the metric presented by [15] (a previous work by the authors). This metric can be categorized as a dynamic metric since has certain dependency on variable network state information (hence, potentially inaccurate) such as link bandwidth and link vulnerability. In Equation (3), $C_{j}^{1(s)}$ is the cost of route $j$ locally computed by a node; the parameter $N_{j}$ is the number of hops of route $j ; \mathcal{L}_{j}^{1(s)}$ is availability of route $j$ computed according to Equation (3); whereas $V_{j}$ is the vulnerability of route $j$, such as $V_{j}^{(s)}=\sum_{i \in j} v_{i}$. Finally, $b_{\min }$

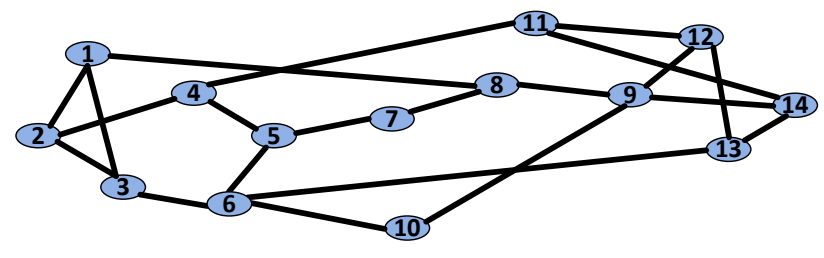

Figure 2. The 14-node, 21 link NSFNet topology.

is the minimum bandwidth available on the links forming route $j$, such as $b_{\min }=\min \left(b_{i}^{(s)}\right) \forall i \in j$. Note that all parameters $\left(V_{j}^{(s)}, b_{\min }, C_{j}^{(s)}\right)$ except $N_{j}$, are locally computed by each node.

$$
C_{j}^{1(s)}=\frac{\mathcal{L}_{j}^{1(s)}}{N_{j}-1}+N_{j}\left(\frac{1}{b_{\min }}\right)\left(V_{j}^{(s)}+1\right)
$$

Since the availability of a route (left term of Equation (3)) usually has more weight than other parameters of Equation (3), we divide it by the route length in order to balance the weight of each parameter. In addition, if a route vulnerability is 0 , HPBR does not rely only on the counter value to compute $C_{j}^{1}$, see the right term of Equation (3). This issue was not addressed by authors in [15].

On the other hand, the other metric used by HPBR (see, Equation (4)) can be categorized as a quasi-permanently metric since it has a low dependency of variable network state information, i.e., it avoids both vulnerability and bandwidth parameters.

In Equation (4), the parameter $\mathcal{L}_{j}^{2(s)}$ is computed in a similar manner as $\mathcal{L}_{j}^{1(s)}$, but the vulnerability degree is not taken into account for its computation, i.e., $\mathcal{L}_{j}^{2(s)}=\sum_{i \in j} p_{i}^{(s)}$.

In addition, we decrease the hysteresis degree of a link counter, such as $p_{i}^{(s)}=p_{i}^{(s)}+2 \Longleftrightarrow$ a connection along link $i$ is blocked and $p_{i}^{(s)}<2$; and $p_{i}^{(s)}=p_{i}^{(s)}-2 \Longleftrightarrow \mathrm{a}$ connection along link $i$ can be successfully provisioned and $p_{i}^{(s)}>0$.

In Section IV, we delve into the issues driving the use of an hybrid metric approach.

$$
C_{j}^{2(s)}=\frac{\mathcal{L}_{j}^{2(s)}}{N_{j}-1}+N_{j}
$$

\section{Model Methodology}

In order to ensure realistic findings, we build up a simulation model of an OTN scenario based on the NSFNet topology (see Fig. 2) using the well-known network simulator OMNeT++ [17]. Each performed trial is the average of three tests using distinct random number generators (RNG). We also adopt two type of dynamic scenarios:

1) Quasi-Incremental or Moderate-Dynamic Scenario. In this scenario the connection requests arrivals (CRA) for each node are as follows: $C R A_{1}\left(t_{1}\right), C R A_{2}\left(t_{1}+\right.$ $\left.t_{2}\right), \ldots C R A_{n}\left(t_{n-1}+t\right)$, such as $t$ is Poissondistributed. 


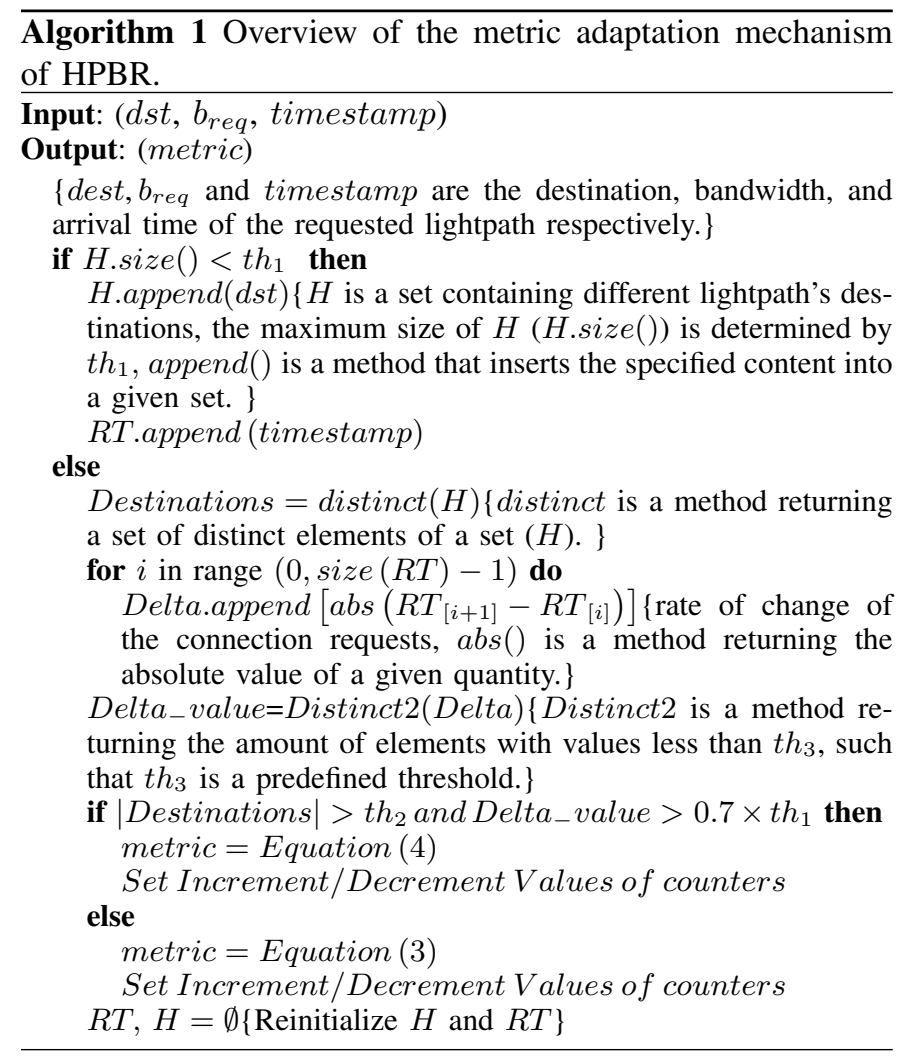

2) Highly-Dynamic Scenario. In this scenario the CRAs for each node are as follows: $C R A_{1}\left(t_{1}\right), C R A_{2}\left(t_{2}\right), \ldots C R A_{n}\left(t_{n}\right)$, such as $t_{n}$ is Poisson-distributed.

In the performed trials the following settings were assumed:

- Both holding time and the average bandwidth requested per connection are Poisson-distributed with an average never exceeding $100 \%$ of the CRA time and $10 \%$ capacity of an optical link respectively.

- Optical nodes with 80 channels on a $50 \mathrm{GHz}$ fixed-grid.

- The evaluated schemes were modeled using the wellknown NSFNet topology.

- In order to reduce path computation complexity a FixedAlternate routing approach was assumed, i.e., 4 precomputed paths per node obtained offline by means of Dijkstra's algorithm.

- The time required by control planes operations such as a connection setup and path computation is neglected.

- Reattempt is not done once a connection is blocked.

- All nodes support wavelength conversion.

- We assume a single fiber system.

- Once a connection is established it cannot be reconfigured during its lifetime.

Based on extensive simulations, the obtained results (presented in Section V) lead us to consider that for Quasi-Incremental scenarios a dynamic metric as the one shown in Equation (3) provides good performance in comparison with other routing schemes. However, for Highly-Dynamic scenarios a quasipermanently metric based on hops, such as the one shown in Equation (4) exhibits a better performance. This is because as the inaccuracy increase -since the available bandwidth is

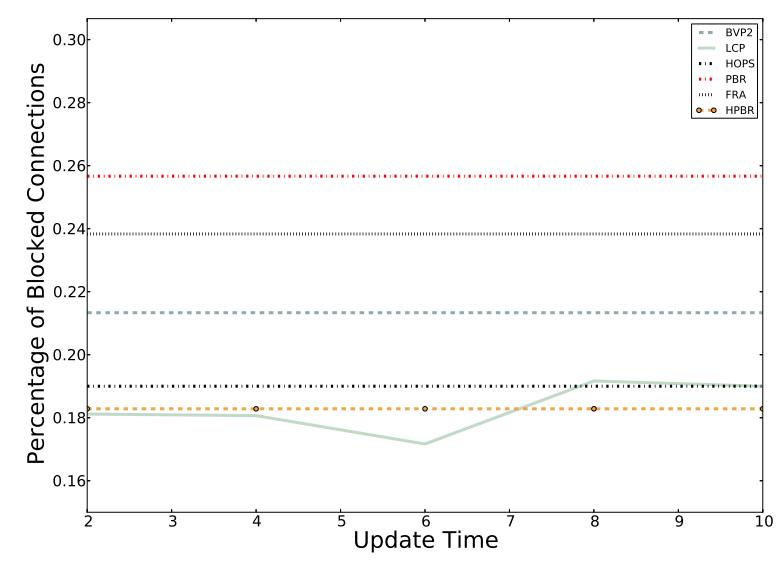

Figure 3. Percentage of blocked connections for a Quasi-Incremental scenario with 100 requests per node, 6 nodes as sources, average holding time and CRA time of 4 units; average $b_{r e q}=10 \%$ of total link capacity; and $\epsilon=5 \%$.

rapidly changing - it is better to rely on routes that span less hops in order to use less bandwidth, and add less inaccuracy. Indeed, our numerical results validate that in Highly-Dynamic scenarios selecting paths that span several hops routes in order to avoid a potential connection blocking is not optimal. Having said so, relying on more network state information may be counterproductive. The performance of static (permanently metric) and dynamic routing has been discussed by [18], [4] Nevertheless, authors do not consider inaccurate and local network state information.

HPBR collects information such as destination and arrival time of CRs in order to evaluate the network conditions. Based on this evaluation HPBR selects either Equation (3) or (4) to compute route costs. The overall procedure of how this is done is elucidated in Algorithm 1. As it can be observed, if a node receives a high amount of CRs during a short period of time (determined by variable Delta_value in Algorithm 1) and the destination nodes (variable Destinations in Algorithm 1) of these requests are highly heterogeneous, then HPBR computes $C_{j}^{(s)}$ based on Equation (4), otherwise it uses Equation (3). In Algorithm 1, th $h_{1}$ determines the amount of collected information; whereas $t h_{2}$ specifies when the condition of CRs with highly-heterogeneous destinations is met; finally, $t h_{3}$ specifies temporal proximity of the CRs. The threshold values of $t h_{1}, t h_{2}$ were set to 20,8 respectively, whereas $t h_{2}$ value was set to 4 units of time.

\section{NUMERICAL RESULTS}

In this section, we present the numerical results related to the proposed scheme namely HPBR and other similar works available in the literature such as PBR, FRA, BVP2 (we adapted BVP2 for OTNs), the well-known Least-Congested Path (LCP), which is only of evaluated schemes that require periodically dissemination of network state information, and HOPS, which uses a routing metric based exclusively on the number of hops along a path.

On one hand, Fig. 3 shows the percentage of blocked connections for a Quasi-Incremental Scenario. In this scenario 


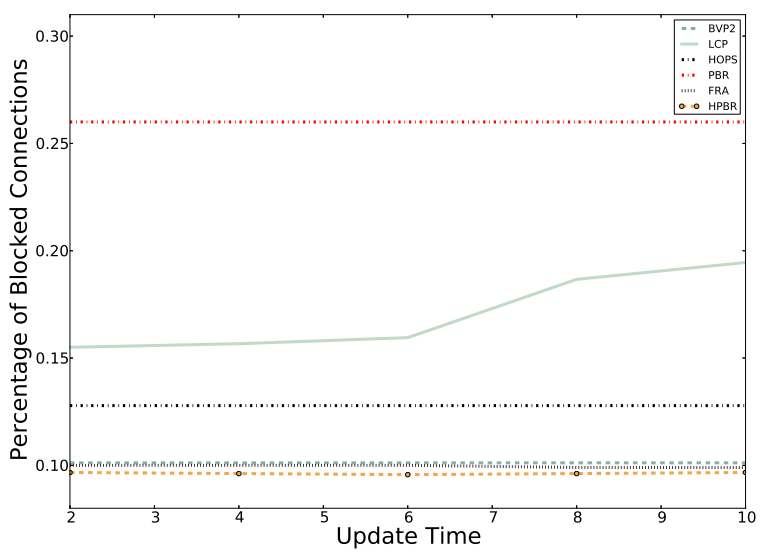

Figure 4. Percentage of blocked connections for a Highly-Dynamic scenario with 100 requests per node, 6 nodes as sources, average holding time and CRA time of 100 units and 10 units respectively; average $b_{r e q}=2 \%$ of total link capacity; and $\epsilon=5 \%$.

LCP and HPBR have a better performance. However, the performance of HPBR is not affected by the update time interval, as it is the case with LCP. This is because in HPBR the dissemination of network state information is only restricted to topological changes.

On the other hand, Fig. 4 shows the percentage of blocked connections for a Highly-Dynamic Scenario. The purpose of this trial is to simulate a network heavily loaded of CRs. For this purpose we select a holding time ten times higher than the CRA time, i.e., increase the number of active connections in the network at any time.

Moreover, Fig. 5 provides numerical results related to a Highly-Dynamic scenario composed of two bursts of requests. The first bust of requests has both holding time and CRA time equal to 10 units of time, whereas the second burst has both holding time and CRA time equal to 30 units of time.

Based on the results obtained from both Fig. 4 and Fig. 5 HPBR presents a better performance compared with its counterparts.

Finally, Fig. 6 presents the numerical results related to a mixture a Quasi-Incremental and a Highly-Dynamic Scenario, where we opt to gradually increase the total of possible destinations -increase the number of active connections at any moment- for a CR. In this scenario, HPBR switches from computing routes as shown Equation (3) to compute routes using Equation (4), once it detects (according to Algorithm 1) that the network conditions entail a different network scenario.

It is important to notice how the performance of a static routing strategy such as HOPS, improves as the inaccuracy degree increases, i.e., more active connections at any moment. This behavior was validated by a similar approach followed by authors in [18]. This motivated us to incorporate an hybrid metric system for HPBR.

Notice that for a low total of distinct destinations FRA shows a low percentage of blocked connections, but for high total of distinct destinations its performance is reduced. The opposite occurs with HOPS scheme. However, HPBR

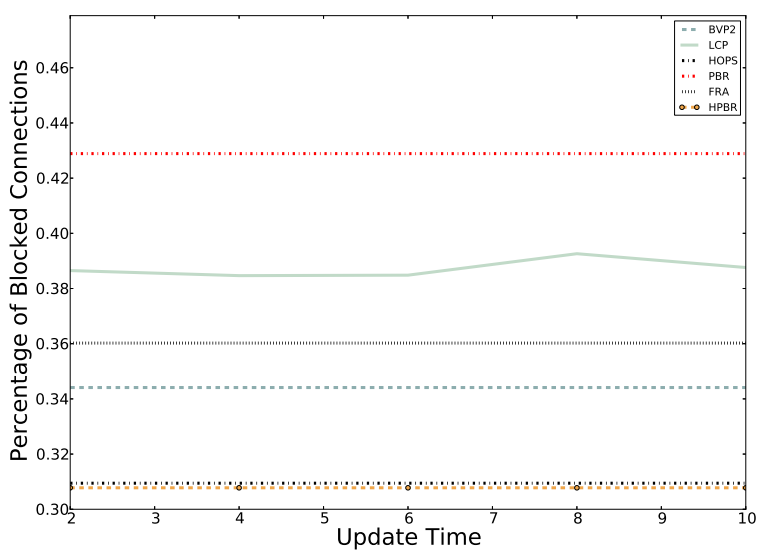

Figure 5. Percentage of blocked connections for a Highly-Dynamic scenario composed by two burst of requests, each burst with 150 requests per node, 6 nodes as sources, average holding time and CRA time of 100 units and 10 units respectively; $b_{r e q}=4 \%$ of total link capacity; and $\epsilon=5 \%$.

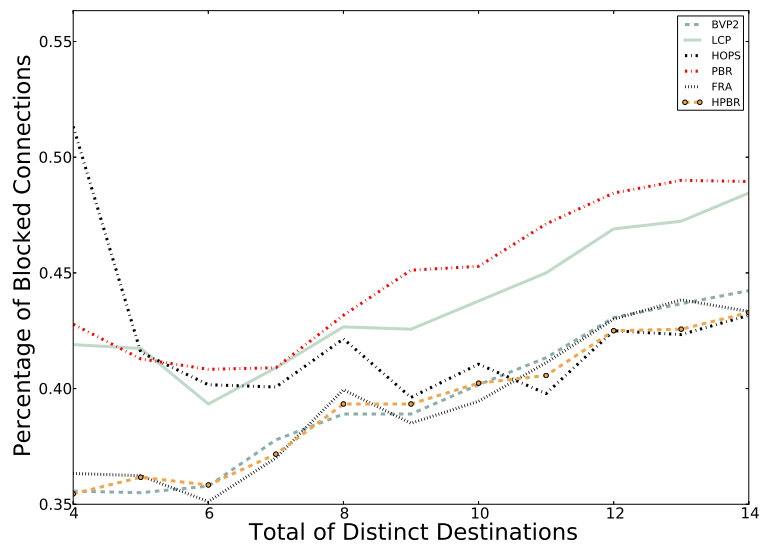

Figure 6. Percentage of blocked connections for a Highly-Dynamic scenario with 200 requests per node, 3 nodes as sources, average holding time and CRA time of 4 units respectively; average $b_{r e q}=4 \%$ of total link capacity; and $\epsilon=5 \%$.

shows low percentage of blocked connections independently of network scenario type. Therefore, it can be stated that HPBR is the best option for addressing the routing inaccuracy problem in both Moderate and Highly-Dynamic scenarios.

\section{CONCLUSIONS}

The contribution of this paper focuses on a novel routing selection algorithm based on prediction techniques referred to as Hybrid Prediction-based Routing (HPBR). HPBR uses two routing metrics according to the network conditions both leveraging prediction techniques in order to deal with the routing inaccuracy problem in Optical Transport Networks (OTNs). The main difference of HPBR compared with similar prediction schemes available in the literature (also dealing with the routing inaccuracy problem) is that it adopts a finergranularity approach for prediction counters for the purpose of predicting a route availability. Obtained results validate that 
the proposed scheme shows a better performance related to the percentage of blocked connections in distinct networks scenarios.

As a future line of work, we intend to study how both the network topology and different blocking factors may impact on the metric type used for routing purposes and the performance of HPBR respectively. In addition, we intend to extend prediction techniques to deal the routing inaccuracy problem considering different routing architectures, such as Path Computation Elements (PCEs).

\section{ACKNOWLEDGMENTS}

This work was supported by the Spanish Ministry of Economy under contract TEC2012-34682, and the Catalan Research Council (CIRIT) under contract 2009 SGR1508.

\section{REFERENCES}

[1] A. Gumaste and N. Krishnaswamy, "Proliferation of the optical transport network: a use case based study," Communications Magazine, IEEE, vol. 48, no. 9, pp. 54-61, 2010.

[2] ITU-T, "Architecture for the Automatically Switched Optical Network (ASON) - Rec. 8080/Y.1304,” 2001.

[3] IETF, "Generalized Multi-Protocol Label Switching (GMPLS) Architecture (RFC 3945),” October 2004.

[4] H. Zang, J. Jue, L. Sahasrabuddhe, R. Ramamurthy, and B. Mukherjee, "Dynamic lightpath establishment in wavelength routed WDM networks," Communications Magazine, IEEE, vol. 39, no. 9, pp. 100-108, 2001.

[5] ITU-T Rec. 68080/4.1304, Architecture for the Automatically Switched Optical Network (ASON)., Std., November 2001.

[6] H. Zang and J. P. Jue, "A review of routing and wavelength assignment approaches for wavelength-routed optical WDM networks," Optical Networks Magazine, vol. 1, pp. 47-60, 2000.

[7] R. A. Guérin, R. A. G. Erin, and A. Orda, "QoS Routing in Networks with Inaccurate Information: Theory and Algorithms," IEEE/ACM Transactions on Networking, 1997.

[8] G. Apostolopoulos, R. Guérin, S. Kamat, and S. K. Tripathi, "Improving QoS Routing Performance Under Inaccurate Link State Information," 1999.

[9] X. Masip-Bruin, S. Sanchez-Lopez, J. Sole-Pareta, and J. DomingoPascual, "QoS routing algorithms under inaccurate routing for bandwidth constrained applications," in Communications, 2003. ICC '03. IEEE International Conference on, vol. 3, 2003, pp. 1743-1748 vol.3.

[10] Y. Luo, Y. Zhang, and W. Gu, "A new method for solving routing and wavelength assignment problems under inaccurate routing information in optical networks with Conversion Capability," in Communications and Photonics Conference and Exhibition (ACP), 2009 Asia, 2009, pp. $1-2$.

[11] S. Shen, G. Xiao, and T. H. Cheng, "The Performance of Periodic Link-State Update in Wavelength-Routed Networks," in Broadband Communications, Networks and Systems, 2006. BROADNETS 2006 3rd International Conference on, 2006, pp. 1-10.

[12] J. Liu, G. Xiao, K. Lu, and I. Chlamtac, "An Evaluation of Distributed Parallel Reservations in Wavelength-Routed Networks," Selected Areas in Communications, IEEE Journal on, vol. 25, no. 9, pp. 27-39, 2007.

[13] E. Marin-Tordera, X. Masip-Bruin, S. Sanchez-Lopez, J. Sole-Pareta, and J. Domingo, "The prediction-based routing in optical transport networks." Computer Networks, vol. 29, pp. 865-878, 2006.

[14] J. E. Smith, "A Study of Branch Prediction Strategies," in Proceedings of the 8th Annual Symposium on Computer Architecture, ser. ISCA '81. Los Alamitos, CA, USA: IEEE Computer Society Press, 1981, pp. 135-148. [Online]. Available: http://dl.acm.org/citation.cfm?id $=800052.801871$

[15] X. Masip-Bruin, E. Marin-Tordera, M. Yannuzzi, R. Serral-Gracia, and S. Sanchez-Lopez, "Reducing the effects of routing inaccuracy by means of prediction and an innovative link-state cost," Communications Letters, IEEE, vol. 14, no. 5, pp. 492-494, 2010.
[16] E. Ahvar, E. Marin-Tordera, M. Yannuzzi, X. Masip-Bruin, and S. Ahvar, "FRA: A new fuzzy-based routing approach for optical transport networks," in Networks and Optical Communications (NOC), 2012 17th European Conference on, 2012, pp. 1-6.

[17] http://www.omnetpp.org/, [Online; accessed February-2014].

[18] A. Zapata-Beghelli and P. Bayvel, "Dynamic Versus Static WavelengthRouted Optical Networks,” Lightwave Technology, Journal of, vol. 26 no. 20, pp. 3403-3415, 2008. 